\title{
LÉVY TYPE CHARACTERIZATION OF STABLE LAWS FOR FREE RANDOM VARIABLES
}

\author{
VITTORINO PATA
}

\begin{abstract}
We give a description of stable probability measures relative to free additive convolution. The definition of domain of attraction is given, and a proof is provided of the noncommutative analogue of Lévy Theorem.
\end{abstract}

\section{INTRODUCTION}

The aim of this paper is to extend some well-known classical results to the case of additive free convolution of probability measures.

The theory is developed in the context of noncommutative probability. Here the notion of independence of random variables is replaced by the notion of freeness; the distribution of a sum of free random variables is the free additive convolution of the distributions. The free additive convolution arises naturally from the noncommutative theory of free products; see $[3,9,10,11]$ for a background of this noncommutative theory.

The paper is organized as follows. Section 2 contains some basic definitions from free probability theory of unbounded random variables. Section 3 presents a review of the relation between the additive convolution and the Cauchy transform of a probability measure. In Section 4 we give the definition of $\boxplus$-infinitely divisible measure (i.e., infinitely divisible with respect to free additive convolution) along with some basic theorems, and we prove that the limit of certain weighted sums of free, identically distributed random variables is $\boxplus$-infinitely divisible. Further we introduce the concept of a stable law. In Section 5 we provide the definition of domain of attraction and we prove that, as in the classical case, a law is stable if and only if it has a non-empty domain of attraction. Finally we focus our attention on weighted sum of free, identically distributed random variables with a $\boxplus$-infinitely divisible distribution $\mu$. Here we show that the Central Limit Theorem holds if and only if $\mu$ has finite variance.

\section{Preliminaries}

We recall some of the basic facts on the theory of free unbounded random variables from [1]. We call a noncommutative probability space a pair $(\mathscr{A}, \varphi)$, where $\mathscr{A}$ is a complex unital noncommutative algebra, and $\varphi: \mathscr{A} \rightarrow \mathbf{C}$ is a linear functional such that $\varphi(1)=1$. If $\mathscr{A}$ is a von Neumann algebra and $\tau$ a

Received by the editors March 18, 1994.

1991 Mathematics Subject Classification. Primary 46L50; Secondary 47C15, 60 E07.

The author was supported by the Consiglio Nazionale delle Ricerche, Italy. 
normal faithful trace, the space $(\mathscr{A}, \tau)$ will be called a noncommutative tracial $W^{*}$-probability space or more simply a $W^{*}$-probability space. In this paper we will always work with a $W^{*}$-probability space $(\mathscr{A}, \tau)$.

A bounded random variable in $(\mathscr{A}, \tau)$ is simply a selfadjoint element of $\mathscr{A}$. An interesting purely noncommutative formal analogue of classical independence is the notion of freeness. The analogy is that around freeness, several concepts can be developed similar to those around independence. A family of von Neumann subalgebras $\left\{\mathscr{A}_{i}\right\}_{i \in I} \subset \mathscr{A}$ in a $W^{*}$-probability space is said to be free if $\tau\left(a_{1} a_{2} \ldots a_{n}\right)=0$ whenever $\tau\left(a_{j}\right)=0, a_{j} \in \mathscr{A}_{i_{j}}$, and $i_{1} \neq i_{2} \neq \ldots \neq i_{n}$. A family of random variables is said to be free if the von Neumann algebras they generate are free. It is interesting to point out that like for classical probability the expectation of the product of two independent random variables is the product of the expectations, here for two free random variables $X, Y \in \mathscr{A}$ we have that $\tau(X Y)=\tau(X) \tau(Y)$.

Using the GNS construction, we may always think of our von Neumann algebra as acting on a Hilbert space $H$. A selfadjoint operator $X$ on $H$ is said to be affiliated with $\mathscr{A}$ if all its spectral projections are in $\mathscr{A}$. More generally a closed densely defined operator $Y$ on $H$ is affiliated with $\mathscr{A}$ if it has a polar decomposition $Y=u X$, with $u \in \mathscr{A}$ and $X$ affiliated with $\mathscr{A}$. Following the notation of [1] we denote the set of all operators on $H$ affiliated with $\mathscr{A}$ by $\widetilde{\mathscr{A}}$, and the subset of selfadjoint operators by $\widetilde{\mathscr{A}}_{s a}$. It is well-known (see [8]) that $\widetilde{\mathscr{A}}$ is an algebra (recall that $\mathscr{A}$ is finite), i.e., if $X, Y \in \widetilde{\mathscr{A}}$ then $X+Y$ and $X Y$ are densely defined, closable, and their closures are in $\widetilde{\mathscr{A}}$. Moreover, if $X, Y \in \widetilde{\mathscr{A}}_{s a}$ then the closure of $X+Y$ is in $\widetilde{\mathscr{A}}_{s a}$.

An unbounded random variable is an element of $\widetilde{\mathscr{A}}_{\underline{s} a}$.

Denote the spectral measure of an operator $X \in \mathscr{A}_{s a}$ by $E_{X}: \mathscr{B}(\mathbf{R}) \rightarrow \mathscr{A}$ (where $\mathscr{B}(\mathbf{R})$ are the Borel sets of the real line $\mathbf{R}$ ), hence $X$ can be written as

$$
X=\int_{-\infty}^{+\infty} \lambda d E_{X}(\lambda)
$$

We say that a family $\left\{X_{i}\right\}_{i \in I}$ of selfadjoint random variables is free if the von Neumann algebras $\left\{\mathscr{A}_{i}\right\}_{i \in I}$ generated by the elements of the form $f\left(X_{i}\right)$, with $f$ a continuous bounded function on $\mathbf{R}$, are free.

Given a $W^{*}$-probability space $(\mathscr{A}, \tau)$ and a random variable $X \in \widetilde{\mathscr{A}}_{s a}$, we define the distribution $\mu_{X}$ of $X$ to be the unique probability measure on $\mathbf{R}$ satisfying the equality $\mu_{X}(\sigma)=\tau\left(E_{X}(\sigma)\right)$, for every $\sigma \in \mathscr{B}(\mathbf{R})$. As in the classical case we define the distribution function $\mathscr{F}_{X}$ as $\mathscr{F}_{X}=\mu_{X}((-\infty, t))$. It is worth recalling that the measure $\mu_{X}$ is compactly supported if and only if $X \in \mathscr{A}$.

An important result is that given a family $\left\{\nu_{i}\right\}_{i \in I}$ of probability measures on $\mathbf{R}$, it is possible to find a $W^{*}$-probability space $(\mathscr{A}, \tau)$ and a family $\left\{X_{i}\right\}_{i \in I}$ of free random variables such that, for all $i \in I \quad \mu_{X_{i}}=\nu_{i}$ (see [1]).

The concept of freeness allows us to define without ambiguity the free additive convolution (indicated by $\boxplus$ ) between two distributions. Indeed, it can be shown that if $X$ and $Y$ are two free random variables then $\mu_{X+Y}$ depends only on $\mu_{X}$ and $\mu_{Y}$, so it is possible to define the operation $\boxplus$ in the following way: $\mu_{X} \boxplus \mu_{Y}=\mu_{X+Y}$. By the above remark, given two probability measures $\mu$ 
and $\nu$, we find a $W^{*}$-probability space $(\mathscr{A}, \tau)$ and two free random variables $X, Y \in \widetilde{\mathscr{A}}_{s a}$ such that $\mu_{X}=\mu$ and $\mu_{Y}=\nu$. Thus it makes sense to define $\mu \boxplus \nu=\mu_{X+Y}$. Therefore the additive convolution is an operation defined on the space of probability measures on $\mathbf{R}$.

\section{AdDitive CONVOLUtion AND $\phi$-FUnCTIONS}

In this section we present some background results from [1] about the analytical method for the calculation of additive free convolution.

Given a probability measure $\mu$ on $\mathbf{R}$, consider the corresponding Cauchy transform

$$
G_{\mu}(z)=\int_{-\infty}^{+\infty} \frac{1}{z-t} d \mu(t), \quad z \notin \mathbf{R} .
$$

Since $G_{\mu}(\bar{z})=\overline{G_{\mu}(z)}$, the behavior of $G_{\mu}$ in the lower complex half-plane $\mathbf{C}^{-}$ is determined by its behavior in the upper half-plane $\mathbf{C}^{+}$. It is clear that $G_{\mu}$ maps $\mathbf{C}^{+}$into $\mathbf{C}^{-}$. It is useful to introduce also the reciprocal of $G_{\mu}$ defined as $F_{\mu}(z)=1 / G_{\mu}(z)$.

We will use the following notation. For positive numbers $\alpha$ and $\beta$ denote

$$
\Gamma_{\alpha}=\{z=x+i y: y>0 \text { and }|x|<\alpha y\},
$$

and

$$
\Gamma_{\alpha, \beta}=\left\{z \in \Gamma_{\alpha}: y>\beta\right\} .
$$

Interest in considering the function $F_{\mu}$ is motivated by the following theorem [1].

Theorem 3.1. Let $\mu$ be a probability measure on $\mathbf{R}$. There exists a domain $\Omega \in \mathbf{C}^{+}$of the form $\Omega=\bigcup_{\alpha>0} \Gamma_{\alpha, \beta(\alpha)}$ such that $F_{\mu}$ has a right inverse $F_{\mu}^{-1}$ defined in $\Omega$, i.e., $F_{\mu}\left(F_{\mu}^{-1}(z)\right)=z$, for $z \in \Omega$. Moreover $\Im F_{\mu}^{-1}(z) \leq \Im z$.

Observe that the function $F_{\mu}^{-1}$ can be extended by symmetry to the domain $\Omega^{*}=\{\bar{\lambda}: \lambda \in \Omega\}$. We are now ready to define the $\phi$-function of a probability measure $\mu$ by

$$
\phi_{\mu}(z)=F_{\mu}^{-1}(z)-z \text {. }
$$

The basic property of the $\phi$-functions is that, given two probability measures $\mu_{1}$ and $\mu_{2}$ it follows that $\phi_{\mu_{1} \boxplus \mu_{2}}=\phi_{\mu_{1}}+\phi_{\mu_{2}}$. Thus the $\phi$-function is the noncommutative analogue of the logarithm of the characteristic function in classical probability. The functions which are $\phi$-functions of probability measure are completely characterized (see [1]). Here we are interested to the continuity properties of the passage $\mu \rightarrow \phi_{\mu}$. For this purpose we state the following result.

Proposition 3.2. Let $\left\{\mu_{n}\right\}_{n=1}^{\infty}$ be a sequence of probability measures on $\mathbf{R}$. The following assertions are equivalent.

(i) The sequence $\left\{\mu_{n}\right\}_{n=1}^{\infty}$ converges weakly to a probability measure $\mu$.

(ii) There exist $\alpha, \beta>0$ such that the sequence $\left\{\phi_{\mu_{n}}\right\}_{n=1}^{\infty}$ converges uniformly on the compact subsets of $\Gamma_{\alpha, \beta}$ to a function $\phi$, and $\phi_{\mu_{n}}(z)=$ $o(z)$ uniformly in $n$ as $|z| \rightarrow \infty, z \in \Gamma_{\alpha, \beta}$.

Moreover, if (i) and (ii) are satisfied, we have $\phi=\phi_{\mu}$ in $\Gamma_{\alpha, \beta}$. 


\section{INFINITELY DIVISIBLE AND STABLE LAWS}

Definition 4.1. A probability measure $\mu$ is said to be $⿴$-infinitely divisible if for every positive integer $n$ there exists a probability measure $\mu_{n}$ such that

$$
\mu=\underbrace{\mu_{n} \boxplus \ldots \boxplus \mu_{n}}_{n \text { times }} .
$$

The $\phi$-function of a $\boxplus$-infinitely divisible measure can be written in a canonical form. This is the content of the next theorem from [1].

Theorem 4.2. The following hold.

(i) A probability measure $\mu$ on $\mathbf{R}$ is $⿴ 囗 十$-infinitely divisible if and only if $\phi_{\mu}$ has an analytic extension defined on $\mathbf{C}^{+}$with values in $\mathbf{C}^{-} \cup \mathbf{R}$.

(ii) Let $\phi: \mathbf{C}^{+} \rightarrow \mathbf{C}^{-}$be an analytic function. Then $\phi$ is a continuation of $\phi_{\mu}$ for some $\boxplus$-infinitely divisible measure $\mu$ if and only if

$$
\lim _{|z| \rightarrow \infty, z \in \Gamma_{\alpha}} \frac{\phi(z)}{z}=0 .
$$

for some (and hence all) $\alpha>0$.

(iii) Let $\mu$ be $a$ 田-infinitely divisible probability measure on $\mathbf{R}$. Then there exist $a \in \mathbf{R}$ and a positive finite measure $\sigma$ such that

$$
\phi_{\mu}(z)=a+\int_{-\infty}^{+\infty} \frac{1+t z}{z-t} d \sigma(t) .
$$

We can better characterize $\boxplus$-infinitely divisible measures with finite variance as follows. The result is an easy consequence of [7].

Proposition 4.3. Let $\mu$ be $a$ 田-infinitely divisible probability measure on $\mathbf{R}$ with $\phi_{\mu}$ given by

$$
\phi_{\mu}(z)=a+\int_{-\infty}^{+\infty} \frac{1+t z}{z-t} d \sigma(t) .
$$

Then $\mu$ has finite variance if and only if $\sigma$ has finite variance.

Proof. Suppose first that $\sigma$ has finite variance. Define

$$
M=\int_{-\infty}^{+\infty} t d \sigma(t)
$$

Then

$$
\phi_{\mu}(z)=b+\int_{-\infty}^{+\infty} \frac{1+t^{2}}{z-t} d \sigma(t),
$$

where $b=a+M$. Then, for every $z=x+i y \in \mathbf{C}^{+}$,

$$
\begin{aligned}
\left|\phi_{\mu}(z)-b\right| & \leq\left|\int_{-\infty}^{+\infty} \frac{1+t^{2}}{z-t} d \sigma(t)\right| \\
& \leq \int_{-\infty}^{+\infty} \frac{1+t^{2}}{|z-t|} d \sigma(t) \leq \int_{-\infty}^{+\infty} \frac{1+t^{2}}{y} d \sigma(t)=\frac{C}{y},
\end{aligned}
$$

where $C<\infty$. Therefore by [7] (cf. Theorem 6.1), $\mu$ has finite variance not exceeding $C$. Conversely suppose that $\mu$ has finite variance. By [7] (cf. 
Theorem 6.2) there exist $\tilde{b} \in \mathbf{R}$ and a positive measure $\tilde{\sigma}$ of finite variance such that

$$
\phi_{\mu}(z)=\tilde{b}+\int_{-\infty}^{+\infty} \frac{1+t^{2}}{z-t} d \tilde{\sigma}(t)
$$

Denote

$$
\tilde{M}=\int_{-\infty}^{+\infty} t d \tilde{\sigma}(t)
$$

Hence

$$
\phi_{\mu}(z)=\tilde{a}+\int_{-\infty}^{+\infty} \frac{1+t z}{z-t} d \tilde{\sigma}(t)
$$

where $\tilde{a}=\tilde{b}-\tilde{M}$. So, by the uniqueness of the Nevanlinna representation, we get that $\tilde{a}=a$ and $\tilde{\sigma}=\sigma$. Therefore $\sigma$ has finite variance.

Another consequence of Theorem 4.2 is the following result.

Proposition 4.4. Let $\left\{\mu_{n}\right\}_{n=1}^{\infty}$ be a sequence of $\boxplus$-infinitely divisible probability measures on $\mathbf{R}$ converging weakly to a probability measure $\mu$. Then $\mu$ is $\boxplus$ infinitely divisible.

Proof. By Proposition 3.2 there exist $\alpha, \beta>0$ such that

$$
\phi_{\mu_{n}}(z) \underset{n \rightarrow \infty}{\longrightarrow} \phi_{\mu}(z),
$$

uniformly on the compact subsets of the truncated cone $\Gamma_{\alpha, \beta}$. By Theorem 4.2, $\phi_{\mu_{n}}$ extends to a function $\psi_{\mu_{n}}: \mathbf{C}^{+} \rightarrow \mathbf{C}^{-} \cup \mathbf{R}$. Since $\left\{\psi_{\mu_{n}}\right\}_{n=1}^{\infty}$ is a normal family, by the Montel Theorem there exists a subsequence $\psi_{\mu_{n_{k}}}$ converging to an analytic function $\psi: \mathbf{C}^{+} \rightarrow \mathbf{C}^{-} \cup \mathbf{R}$. It follows that $\phi_{\mu}$, being the restriction of $\psi$ on $\Gamma_{\alpha, \beta}$ equal to $\phi_{\mu}$, has an analytic extension on $\mathbf{C}^{+}$with values in $\mathbf{C}^{-} \cup \mathbf{R}$. Therefore, using again Theorem 4.2, the result is proved.

We want now to begin a discussion, which will be developed further in the next section, about the properties of limit distributions of certain averages of free, identically distributed (f.i.d.) random variables. Notice that if $X$ is a random variable in a $W^{*}$-probability space, and $c$ is a positive constant, then

$$
\phi_{\mu_{c X}}(z)=c \phi_{\mu_{X}}\left(\frac{z}{c}\right) \text {. }
$$

Proposition 4.5. Let $\left\{X_{n}\right\}_{n=1}^{\infty}$ be a sequence of f.i.d. random variables in a $W^{*}$ probability space with common distribution $\nu,\left\{A_{n}\right\}_{n=1}^{\infty}$ and $\left\{B_{n}\right\}_{n=1}^{\infty}$ two sequences of real and positive numbers, respectively. If the distribution of the random variable $B_{n}^{-1}\left(X_{1}+\ldots+X_{n}\right)-A_{n}$ converges weakly to a probability measure $\mu$, then $\mu$ is $\boxplus$-infinitely divisible.

Proof. Define

$$
Z_{n}=\frac{X_{1}+\ldots+X_{n}}{B_{n}}-A_{n},
$$

and denote by $\psi_{n}$ the $\phi$-function of $Z_{n}$. First we prove that

$$
\lim _{n \rightarrow \infty} B_{n}=\infty \text {. }
$$

Indeed, by Proposition 3.2, there exist $\alpha, \beta>0$ such that

$$
\psi_{n}(z)=\frac{n}{B_{n}} \phi_{\nu}\left(B_{n} z\right)-A_{n} \underset{n \rightarrow \infty}{\longrightarrow} \phi_{\mu}(z)
$$


uniformly on the compact subsets of the truncated cone $\Gamma_{\alpha, \beta}$. If there exists a subsequence $B_{n_{i}} \rightarrow B<\infty$, then, for every fixed $z \in \Gamma_{\alpha, \beta}$,

$$
\left|\Im \psi_{n_{i}}(z)\right|=\left|\Im\left(\frac{n_{i}}{B_{n_{i}}} \phi_{\nu}\left(B_{n_{i}} z\right)\right)\right| \underset{i \rightarrow \infty}{\longrightarrow} \infty .
$$

So the assertion is proved. Notice further that $\phi_{\nu}$ is defined in

$$
\Omega=\bigcup_{\omega>0} \Gamma_{\omega, \theta(\omega)}
$$

So if we set

we get that $\psi_{n}$ is defined in

$$
\theta(\omega, n)=\frac{\theta(\omega)}{B_{n}}
$$

$$
\Omega_{n}=\bigcup_{\omega>0} \Gamma_{\omega, \theta(\omega, n)} .
$$

Note that $\Omega_{n} \subset \Omega_{n+1}$, and

$$
\bigcup_{n=1}^{\infty} \Omega_{n}=\mathbf{C}^{+}
$$

Let $\Phi_{k}=\left\{\psi_{n}\right\}_{n \geq k}$. For every $k$ the family $\Phi_{k}$ is normal, thus by the Montel Theorem there exists a subsequence $\psi_{n_{i}}$ (with $n_{i}=n_{i}(k) \geq k$ ) converging to an analytic function $\psi^{(k)}$ uniformly on the compact subsets of $\Omega_{k}$.

Since $\psi_{n_{i}} \rightarrow \phi_{\mu}$ uniformly on the compact subsets of $\Gamma_{\alpha, \beta}$, by the Identity Theorem it follows that $\phi_{\mu}$ extends to $\Omega_{k}$. Since this holds for every $k, \phi_{\mu}$ has an analytic extension on $\mathbf{C}^{+}$with values in $\mathbf{C}^{-} \cup \mathbf{R}$. Hence, by Theorem 4.2, $\mu$ is $\boxplus$-infinitely divisible.

Definition 4.6. Let $\mu$ and $\nu$ be probability measures on $\mathbf{R}$. We say that $\nu$ and $\mu$ are equivalent $(\mu \sim \nu)$ if there exist constants $a>0$ and $b \in \mathbf{R}$ such that $\mathscr{F}_{\nu}(x)=\mathscr{F}_{\mu}(a x+b)$ for all $x$. By analogy with classical probability, we say that $\mu$ is stable relative to additive free convolution (or simply stable) if $\mu \sim \nu \boxplus \nu^{\prime}$ whenever $\mu \sim \nu \sim \nu^{\prime}$.

It is easy to verify that stable probability distributions are $\boxplus$-infinitely divisible. Moreover if $\mu$ and $\nu$ are probability measures on $\mathbf{R}$, then the following two facts are equivalent:

(i) $\mathscr{F}_{\nu}(x)=\mathscr{F}_{\mu}(a x+b)$ for all $x \in \mathbf{R}$,

(ii) $\phi_{\nu}(z)=a^{-1}\left(\phi_{\mu}(a z)-b\right)$ for all $z \in \mathbf{C}^{+}$,

where $a>0$ and $b \in \mathbf{R}$.

Definition 4.7. Let $\phi: \mathbf{C}^{+} \rightarrow \mathbf{C}^{-} \cup \mathbf{R}$ be an analytic function. We say that $\phi$ is stable if for every number $a>0$ there exist $b>0$ and $c \in \mathbf{R}$ such that

$$
\phi(z)+a \phi\left(\frac{z}{a}\right)=b \phi\left(\frac{z}{b}\right)+c .
$$

Note that it is enough to require that the above equality holds for every $a \geq 1$.

The following corollary is a direct consequence of the above remarks. 
Corollary 4.8. $A \boxplus$-infinitely divisible probability measure $\mu$ is stable if and only if the function $\phi_{\mu}$ is stable.

The next result, whose proof may be found in [1], gives a complete characterization of stable analytic functions (and hence of stable laws).

Theorem 4.9. The following is a complete list of the stable analytic functions $\phi: \mathbf{C}^{+} \rightarrow \mathbf{C}^{-} \cup \mathbf{R}$.

(i) $\phi(z)=a, a \in \mathbf{R}$.

(ii) $\phi(z)=a+i b, a \in \mathbf{R}$ and $b<0$.

(iii) $\phi(z)=a+b z^{-\alpha+1}, a \in \mathbf{R}, \alpha \in(1,2], b \neq 0$, and $\arg b \in$ $[(\alpha-2) \pi, 0]$.

(iv) $\phi(z)=a+b z^{-\alpha+1}, a \in \mathbf{R}, \alpha \in(0,1), b \neq 0$, and $\arg b \in[\pi$, $(1+\alpha) \pi]$.

(v) $\phi(z)=a+b \log z, a \in \mathbf{C}^{-} \cup \mathbf{R}$ and $b<0$.

In the sequel we will refer to $\alpha$ as the exponent of the stable law. A stable law of exponent 1 has a $\phi$-function of type (i), (ii) or (v).

\section{Domains of AtTRACTION}

Definition 5.1. Let $\left\{X_{n}\right\}_{n=1}^{\infty}$ be a sequence of f.i.d. random variables with common distribution $\nu$ in a $W^{*}$-probability space and let $\left\{A_{n}\right\}_{n=1}^{\infty}$ and $\left\{B_{n}\right\}_{n=1}^{\infty}$ be two sequences of real and positive numbers, respectively. Set

$$
Z_{n}=\frac{X_{1}+\ldots+X_{n}}{B_{n}}-A_{n}
$$

If for a suitable choice of the constants $B_{n}$ and $A_{n}$ the distribution of $Z_{n}$ converges weakly to a non-degenerate measure $\mu$ (i.e., a measure not concentrated in one point), we say that $\nu$ is attracted to $\mu$. The set of all probability measures attracted to $\mu$ is called the domain of attraction of $\mu$.

The exclusion of degenerate measures serves to eliminate the trivial situation where $A_{n}$ converges to a finite number while $B_{n}$ increases so rapidly that the random variable $B_{n}^{-1}\left(X_{1}+\ldots+X_{n}\right)$ tends in distribution to zero. We already know from Proposition 4.5 that a probability measure with a non-empty domain of attraction is $\boxplus$-infinitely divisible. Our main result, which is the formal analogue of the classical theorem proved by Lévy (see $[4,5]$ ), is expressed in the following theorem.

Theorem 5.2. A non-degenerate probability measure has a non-empty domain of attraction if and only if it is a stable law.

We first establish the following lemma.

Lemma 5.3. Let $\left\{X_{n}\right\}_{n=1}^{\infty}$ be a sequence of f.i.d. random variables in a $W^{*}$ probability space with common distribution $\nu,\left\{A_{n}\right\}_{n=1}^{\infty}$ and $\left\{B_{n}\right\}_{n=1}^{\infty}$ two sequences of real and positive numbers, respectively. If the distribution of the random variable $B_{n}^{-1}\left(X_{1}+\ldots+X_{n}\right)-A_{n}$ converges weakly to a non-degenerate 
probability measure $\mu$, then

Proof. Define

$$
\begin{aligned}
& \text { (i) } \lim _{n \rightarrow \infty} B_{n}=\infty . \\
& \text { (ii) } \lim _{n \rightarrow \infty} \frac{B_{n+1}}{B_{n}}=1 .
\end{aligned}
$$

$$
Z_{n}=\frac{X_{1}+\ldots+X_{n}}{B_{n}}-A_{n},
$$

and denote by $\mu_{n}$ the distribution of $Z_{n}$. By Proposition 3.2 there exist $\alpha, \beta>$ 0 such that

$$
\phi_{\mu_{n}}(z)=\frac{n}{B_{n}} \phi_{\nu}\left(B_{n} z\right)-A_{n} \underset{n \rightarrow \infty}{\longrightarrow} \phi_{\mu}(z),
$$

uniformly on the compact subsets of the truncated cone $\Gamma_{\alpha, \beta}$.

Assertion (i) follows from the proof of Proposition 4.5. To show (ii) observe that

and

$$
Z_{n+1}=\frac{B_{n}}{B_{n+1}} Z_{n}+\frac{X_{n+1}}{B_{n+1}}+\left(\frac{A_{n} B_{n}}{B_{n+1}}-A_{n+1}\right),
$$

$$
Z_{n}+\frac{X_{n+1}}{B_{n}}=\frac{B_{n+1}}{B_{n}} Z_{n+1}+\left(\frac{A_{n+1} B_{n+1}}{B_{n}}-A_{n}\right) .
$$

By assertion (i) and Proposition 3.2, there exist $\alpha^{\prime}, \beta^{\prime}>0$ such that

$$
\Im\left(\frac{B_{n}}{B_{n+1}} \phi_{\mu_{n}}\left(\frac{B_{n+1}}{B_{n}} z\right)\right) \underset{n \rightarrow \infty}{\longrightarrow} \phi_{\mu}(z),
$$

and

$$
\Im\left(\frac{B_{n+1}}{B_{n}} \phi_{\mu_{n+1}}\left(\frac{B_{n}}{B_{n+1}} z\right)\right) \underset{n \rightarrow \infty}{\longrightarrow} \Im \phi_{\mu}(z),
$$

uniformly on the compact subsets of $\Gamma_{\alpha^{\prime}, \beta^{\prime}} \subset \Gamma_{\alpha, \beta}$.

Applying Proposition 3.2 once more to $\phi_{\mu_{n}}$ we see that $B_{n+1} / B_{n}$ is bounded away from zero and infinity. Indeed, suppose that $B_{n+1} / B_{n}$ is not bounded away, for example, from zero. Then, provided that we pass to a subsequence, we have that, for every fixed $z$ in the truncated cone $\Gamma_{\alpha^{\prime}, \beta^{\prime}}$, the left-hand side of the second equation goes to zero. Hence there exists $N>1$ such that $B_{n+1} / B_{n} \in[1 / N, N]$ for every $n$.

Let us denote throughout the rest of the proof

$$
c_{n}=\frac{B_{n+1}}{B_{n}} .
$$

Selecting $z=i y$, with $y$ big enough, we get

$$
z c_{n} \in\left[\frac{i y}{N}, i y N\right] \subset \Gamma_{\alpha^{\prime}, \beta^{\prime}} .
$$

Thus for each $\varepsilon>0$ there exists $n_{0}(\varepsilon) \in \mathbf{N}$ such that, for $n \geq n_{0}(\varepsilon)$,

$$
\left|\Im \phi_{\mu_{n}}\left(c_{n} z\right)-\Im \phi_{\mu}\left(c_{n} z\right)\right|<\frac{\varepsilon}{2 N},
$$


from which it follows that

$$
\left|\Im\left(\frac{1}{c_{n}} \phi_{\mu_{n}}\left(c_{n} z\right)\right)-\Im\left(\frac{1}{c_{n}} \phi_{\mu}\left(c_{n} z\right)\right)\right|<\frac{\varepsilon}{2 N c_{n}} \leq \frac{\varepsilon}{2},
$$

and

$$
\left|\Im\left(\frac{1}{c_{n}} \phi_{\mu_{n}}\left(c_{n} z\right)\right)-\Im \phi_{\mu}(z)\right|<\frac{\varepsilon}{2} .
$$

Therefore, combining the last two inequalities, we get that, for $n \geq n_{0}(\varepsilon)$,

$$
\left.\mid \Im\left(\frac{1}{c_{n}} \phi_{\mu}\left(c_{n} z\right)\right)-\Im \phi_{\mu}(z)\right) \mid<\varepsilon .
$$

By Proposition $4.5 \mu$ is $\boxplus$-infinitely divisible and, since we suppose $\mu$ nondegenerate, by Theorem 4.2 there exists a positive measure $\sigma \neq 0$ and $a \in \mathbf{R}$ such that

$$
\phi_{\mu}(z)=a+\int_{-\infty}^{+\infty} \frac{1+t z}{z-t} d \sigma(t) .
$$

Therefore

$$
\Im \phi_{\mu}(i y)=-\int_{-\infty}^{+\infty} \frac{y\left(1+t^{2}\right)}{t^{2}+y^{2}} d \sigma(t),
$$

and we get

$$
\begin{aligned}
& \left.\mid \Im\left(\frac{1}{c_{n}} \phi_{\mu}\left(c_{n} z\right)\right)-\Im \phi_{\mu}(z)\right) \mid \\
& \quad=y\left|\int_{-\infty}^{+\infty}\left(1+t^{2}\right)\left(\frac{1}{t^{2}+c_{n}^{2} y^{2}}-\frac{1}{t^{2}+y^{2}}\right) d \sigma(t)\right| \\
& \quad=y^{3}\left|1-c_{n}^{2}\right| \int_{-\infty}^{+\infty} \frac{1+t^{2}}{\left(t^{2}+c_{n}^{2} y^{2}\right)\left(t^{2}+y^{2}\right)} d \sigma(t) \\
& \quad \geq y^{3}\left|1-c_{n}^{2}\right| \int_{-\infty}^{+\infty} \frac{1+t^{2}}{\left(t^{2}+N^{2} y^{2}\right)\left(t^{2}+y^{2}\right)} d \sigma(t)
\end{aligned}
$$

Since, once $y$ is fixed,

$$
y^{3} \int_{-\infty}^{+\infty} \frac{1+t^{2}}{\left(t^{2}+N^{2} y^{2}\right)\left(t^{2}+y^{2}\right)} d \sigma(t)=C \geq 0,
$$

it follows that for $n \geq n_{0}(\varepsilon)$,

$$
\left|1-c_{n}^{2}\right| \leq \frac{\varepsilon}{C}
$$

Thus, letting $\varepsilon \rightarrow 0$, we finally get

$$
\lim _{n \rightarrow \infty} c_{n}=1
$$

which yields the result.

Proof of Theorem 5.2. Necessity. Let $\mu$ be a non-degenerate probability measure. Let $\left\{X_{n}\right\}_{n=1}^{\infty}$ be a sequence of f.i.d. random variables in a $W^{*}$-probability space, $\left\{A_{n}\right\}_{n=1}^{\infty}$ and $\left\{B_{n}\right\}_{n=1}^{\infty}$ two sequences of real and positive numbers, respectively, such that $Z_{n}$ (defined as in Lemma 5.3), converges in distribution 
to $\mu$. Denote by $\mu_{n}$ the distribution of $Z_{n}$. Select $a \geq 1$. By Lemma 5.3 it is possible to find a sequence $m=m(n)$ such that

$$
\lim _{n \rightarrow \infty} \frac{B_{m(n)}}{B_{n}}=a
$$

and

$$
\lim _{n \rightarrow \infty} m(n)=\infty
$$

Define

$$
Z_{m}^{\prime}=\frac{X_{n+1}+\ldots+X_{n+m}}{B_{m}}-A_{m}
$$

It is clear that $Z_{m}$ and $Z_{m}^{\prime}$ have the same distribution. Let us consider the sum

$$
Z_{n}+\frac{B_{m}}{B_{n}} Z_{m}^{\prime}=\frac{X_{1}+\ldots+X_{n+m}}{B_{n}}-H_{n}
$$

where

$$
H_{n}=A_{n}+\frac{B_{m}}{B_{n}} A_{m}
$$

From the assumptions of the theorem, the distribution functions of the two components of the left-hand side converge to $\mathscr{F}_{\mu}(x)$ and $\mathscr{F}_{\mu}(x / a)$, respectively. Therefore, by Proposition 3.2, there exists a truncated cone $\Gamma_{\alpha, \beta}$ such that the $\phi$-function of the right-hand side converges to $\phi_{\mu}(z)+a \phi_{\mu}(z / a)$ and $\phi_{\mu_{n}}(z)$ converges to $\phi_{\mu}(z)$ uniformly on the compact subsets of $\Gamma_{\alpha, \beta}$.

Moreover

$$
\frac{X_{1}+\ldots+X_{n+m}}{B_{n}}-H_{n}=\frac{B_{n+m}}{B_{n}} Z_{n+m}+K_{n},
$$

where

$$
K_{n}=\frac{B_{n+m}}{B_{n}} A_{n+m}-H_{n}
$$

Hence, setting

$$
a_{n}=\frac{B_{m}}{B_{n}},
$$

and

$$
b_{n}=\frac{B_{n+m}}{B_{n}} \text {, }
$$

we get

$$
b_{n} \phi_{\mu_{n}}\left(\frac{z}{b_{n}}\right)+K_{n} \underset{n \rightarrow \infty}{\longrightarrow} \phi_{\mu}(z)+a \phi_{\mu}\left(\frac{z}{a}\right),
$$

uniformly on the compact subsets of $\Gamma_{\alpha, \beta}$. On the other hand,

$$
Z_{n+m}=\frac{B_{n}}{B_{n+m}} Z_{n}+\frac{B_{n}}{B_{n+m}} \frac{B_{m}}{B_{n}} Z_{m}^{\prime}+I_{n},
$$

where

$$
I_{n}=\frac{B_{n}}{B_{n+m}} A_{n}+\frac{B_{m}}{B_{n+m}} A_{m}-A_{n+m} .
$$

Hence, using the above notation,

$$
Z_{n+m}=\frac{1}{b_{n}} Z_{n}+\frac{a_{n}}{b_{n}} Z_{m}^{\prime}+I_{n} .
$$


Thus it follows that

$$
\frac{1}{b_{n}} \phi_{\mu_{n}}\left(b_{n} z\right)+\frac{a_{n}}{b_{n}} \phi_{\mu_{m}}\left(\frac{b_{n}}{a_{n}} z\right)+I_{n} \underset{n \rightarrow \infty}{\longrightarrow} \phi_{\mu}(z),
$$

uniformly on the compact subsets of $\Gamma_{\alpha, \beta}$. In particular, for every $z \in \Gamma_{\alpha, \beta}$, we have

$$
\Im\left(b_{n} \phi_{\mu_{n}}\left(\frac{z}{b_{n}}\right)\right) \underset{n \rightarrow \infty}{\longrightarrow} \Im \phi_{\mu}(z)+\Im\left(a \phi_{\mu}\left(\frac{z}{a}\right)\right),
$$

and

$$
\Im\left(\frac{1}{b_{n}} \phi_{\mu_{n}}\left(b_{n} z\right)+\frac{a_{n}}{b_{n}} \phi_{\mu_{m}}\left(\frac{b_{n}}{a_{n}} z\right)\right) \underset{n \rightarrow \infty}{\longrightarrow} \Im \phi_{\mu}(z) .
$$

So, using the same argument of Lemma 5.3, and recalling that $a_{n}$ converges to $a$, we get that $b_{n}$ is bounded away from zero and infinity. Moreover the two equations above tell us that in fact $b_{n}$ must converge to a limit $b$. This forces $K_{n}$ to converge to a finite limit $K$. By Proposition 3.2, we have

$$
b_{n} \phi_{\mu_{n}}\left(\frac{z}{b_{n}}\right)+K_{n} \underset{n \rightarrow \infty}{\longrightarrow} b \phi_{\mu}\left(\frac{z}{b}\right)+K
$$

in some truncated cone $\Gamma_{\alpha^{\prime}, \beta^{\prime}} \subset \Gamma_{\alpha, \beta}$, and by the Identity Theorem we finally get

$$
\phi_{\mu}(z)+a \phi_{\mu}\left(\frac{z}{a}\right)=b \phi_{\mu}\left(\frac{z}{b}\right)+K,
$$

for every $z \in \mathbf{C}^{+}$. Therefore, by Corollary $4.8, \mu$ is stable.

Sufficiency. Let $\mu$ be stable, and let $\left\{X_{n}\right\}_{n=1}^{\infty}$ be a sequence of f.i.d. random variables with common distribution $\mu$. Then there exist sequences $\left\{A_{n}\right\}_{n=1}^{\infty}$ and $\left\{B_{n}\right\}_{n=1}^{\infty}$ of real and positive numbers, respectively, such that the sum $X_{1}+$ $\ldots+X_{n}$, has distribution function $\mathscr{F}_{\mu}\left(B_{n}^{-1} x-A_{n}\right)$. Hence the random variable $Z_{n}$, defined by $Z_{n}=B_{n}^{-1}\left(X_{1}+\ldots+X_{n}\right)-A_{n}$, has distribution function $\mathscr{F}_{\mu}(x)$. Thus $\mu$ belongs to its own domain of attraction.

Our next goal is to give a precise characterization of the coefficients $B_{n}$ which appear in the weighted sum. For this purpose we need first a definition.

Definition 5.4. A function $h$ of integer argument is said to be slowly varying if for any integer $k$

$$
\lim _{n \rightarrow \infty} \frac{h(k n)}{h(n)}=1 \text {. }
$$

Theorem 5.5. Let $\left\{X_{n}\right\}_{n=1}^{\infty}$ be a sequence of f.i.d. random variables in a $W^{*}$-probability space, $\left\{A_{n}\right\}_{n=1}^{\infty}$ and $\left\{B_{n}\right\}_{n=1}^{\infty}$ two sequences of real and positive numbers, respectively. If the distribution of $B_{n}^{-1}\left(X_{1}+\ldots+X_{n}\right)-A_{n}$ converges weakly to a non-degenerate probability measure $\mu$, then $B_{n}=n^{\frac{1}{*}} h(n)$, with $\alpha \in(0,2]$ and $h$ a slowly varying function.

Proof. Let $m$ be a positive integer. Define for every $i=0,1, \ldots, m-1$

$$
S_{n}^{i}=\frac{X_{n i+1}+\ldots+X_{n(i+1)}}{B_{n}}
$$

and

$$
Z_{n}=S_{n}^{0}-A_{n}
$$


It is clear that for every $i=0,1, \ldots, m-1$ the random variables $S_{n}^{i}$ are free and have the same distribution. Denote by $\nu_{n}$ the common distribution of $S_{n}^{i}$ and by $\mu_{n}$ the distribution of $Z_{n}$. Then, denoting

$$
c_{n}=c_{n}(m)=\frac{B_{n m}}{B_{n}}
$$

we get

$$
S_{n m}^{0}=\frac{1}{c_{n}}\left(S_{n}^{0}+\ldots+S_{n}^{m-1}\right)
$$

which yields

$$
\phi_{\nu_{n m}}(z)=\frac{m}{c_{n}} \phi_{\nu_{n}}\left(c_{n} z\right)
$$

On the other hand, we may write the former relation as

$$
S_{n}^{0}+\ldots+S_{n}^{m-1}=c_{n} S_{n m}^{0}
$$

which yields

$$
m \phi_{\nu_{n}}(z)=c_{n} \phi_{\nu_{n m}}\left(\frac{z}{c_{n}}\right) .
$$

Again, as in Lemma 5.3 we get that $c_{n}$ must converge to a finite limit $c$. Since for every $n$ the equality $\Im \phi_{\nu_{n}}(z)=\Im \phi_{\mu_{n}}(z)$ holds for any $z$ in certain truncated cone, it follows that, for $z$ big enough, $z$ in the truncated cone,

$$
\Im \phi_{\mu}(z)=\Im\left(\frac{m}{c} \phi_{\mu}(c z)\right) .
$$

This equality, by the Identity Theorem (for harmonic functions), holds for every $z \in \mathbf{C}^{+}$. Since $\mu$ is stable and non-degenerate, its $\phi$-function is given by (ii), (iii), (iv) or (v) of Theorem 4.9. In any of these four cases, it follows directly by the above relation that $c=m^{\frac{1}{\alpha}}$ with $\alpha \in(0,2]$, i.e.,

$$
\lim _{n \rightarrow \infty} \frac{B_{n m}}{B_{n}}=m^{\frac{1}{\alpha}}
$$

which is possible only if

$$
B_{n}=n^{\frac{1}{\alpha}} h(n)
$$

with $h$ slowly varying.

It is immediate to verify that if $\nu$ and $\omega$ are equivalent non-degenerate stable laws, they have the same domain of attraction. The converse is also true.

Theorem 5.6. Domains of attraction of non-degenerate non-equivalent stable laws are disjoint.

Proof. Suppose that $\nu$ and $\omega$ are two non-degenerate stable laws with the same domain of attraction. Then there exist $\alpha, \beta \in(0,2], h$ and $h^{\prime}$ slowly varying functions, $\left\{A_{n}\right\}_{n=1}^{\infty}$ and $\left\{A_{n}^{\prime}\right\}_{n=1}^{\infty}$ sequences of real numbers, and a sequence 
$\left\{X_{n}\right\}_{n=1}^{\infty}$ of f.i.d. random variables such that, defining

$$
Z_{n}=\frac{X_{1}+\ldots+X_{n}}{h(n) n^{\frac{1}{\alpha}}}-A_{n}
$$

and

$$
Z_{n}^{\prime}=\frac{X_{1}+\ldots+X_{n}}{h^{\prime}(n) n^{\frac{1}{\beta}}}-A_{n}^{\prime},
$$

it follows that, for every $z$ in some truncated cone,

$$
\phi_{\mu_{Z_{n}}}(z) \underset{n \rightarrow \infty}{\longrightarrow} \phi_{\nu}(z)
$$

and

$$
\dot{\phi}_{\mu_{z_{n}^{\prime}}}(z) \underset{n \rightarrow \infty}{\longrightarrow} \phi_{\omega}(z)
$$

We first show that we necessarily have $\alpha=\beta$. Suppose indeed that $\alpha>\beta$. Then

$$
Z_{n}^{\prime}=a_{n} Z_{n}+a_{n} A_{n}-A_{n}^{\prime},
$$

where

$$
a_{n}=\frac{h(n) n^{\frac{1}{\alpha}}}{h^{\prime}(n) n^{\frac{1}{\beta}}} \underset{n \rightarrow \infty}{\longrightarrow} 0 .
$$

Therefore by Proposition 3.2, for every $z$ in the truncated cone we have

$$
\Im\left(a_{n} \phi_{\mu_{Z_{n}}}\left(\frac{z}{a_{n}}\right)\right) \underset{n \rightarrow \infty}{\longrightarrow} 0 .
$$

On the other hand we have also that

$$
\Im\left(a_{n} \phi_{\mu_{z_{n}}}\left(\frac{z}{a_{n}}\right)\right) \underset{n \rightarrow \infty}{\longrightarrow} \Im \phi_{\omega}(z),
$$

which is a contradiction. Hence $\alpha=\beta$.

Defining now

$$
k_{n}=\frac{h(n)}{h^{\prime}(n)},
$$

we have that

$$
\Im\left(k_{n} \phi_{\mu_{z_{n}}}\left(\frac{z}{k_{n}}\right)\right) \underset{n \rightarrow \infty}{\longrightarrow} \Im \phi_{\omega}(z),
$$

and

$$
\Im\left(\frac{1}{k_{n}} \phi_{\mu_{z_{n}^{\prime}}}\left(k_{n} z\right)\right) \underset{n \rightarrow \infty}{\longrightarrow} \Im \phi_{\nu}(z),
$$

for every $z$ in some truncated cone. Again, as in Lemma 5.3, we get that $k_{n}$ must converge to a finite positive limit $k$. By the Identity Theorem then, for every $z \in \mathbf{C}^{+}$, we get

$$
\Im\left(k \phi_{\nu}\left(\frac{z}{k}\right)\right)=\Im \phi_{\omega}(z) .
$$

For $\alpha=2$ there is just one class of equivalent stable laws, thus there is nothing to prove. For $\alpha=1$ there are two classes of equivalent stable laws, namely 
(according to the classification in Theorem 4.9), stable laws of type (ii) and (v). So if $\nu$ is of type (ii) and $\omega$ is of type ( $v)$, Equation ( $*$ ) becomes

$$
k b_{1}=b_{2} \Im(\log z), \quad z \in \mathbf{C}^{+},
$$

and this is a contradiction. Finally for $\alpha \in(0,1) \cup(1,2)$ Equation $(*)$ leads to

$$
k^{\alpha} \Im\left(k b_{1} z^{-\alpha+1}\right)=\Im\left(b_{2} z^{-\alpha+1}\right), \quad z \in \mathbf{C}^{+},
$$

which is possible if and only if $\arg \left(b_{1}\right)=\arg \left(b_{2}\right)$, i.e., if and only if $\nu$ and $\omega$ are equivalent.

The stable law of exponent $\alpha=2$ is the semicircle law, defined as

$$
d \gamma_{a, r}(t)= \begin{cases}\frac{2}{\pi r^{2}} \sqrt{r^{2}-(t-a)^{2}} d t & \text { if } t \in[a-r, a+r] \\ 0 & \text { otherwise }\end{cases}
$$

which is the noncommutative analogue of the normal law. Indeed, an easy calculation shows that

$$
\phi_{\gamma_{a, r}}(z)=a+\frac{r^{2}}{4} \frac{1}{z}
$$

from where we get

$$
\gamma_{a_{1}, r_{1}} \boxplus \gamma_{a_{2}, r_{2}}=\gamma_{a_{1}+a_{2}, \sqrt{r_{1}^{2}+r_{2}^{2}}}
$$

Note that

$$
\operatorname{Mean}\left(\gamma_{a, r}\right)=\int_{-\infty}^{+\infty} t d \gamma_{a, r}(t)=a
$$

and

$$
\operatorname{Var}\left(\gamma_{a, r}\right)=\int_{-\infty}^{+\infty}(t-a)^{2} d \gamma_{a, r}(t)=\frac{r^{2}}{4}
$$

In the sequel, we will denote $\gamma_{0, r}$ by $\gamma_{r}$.

We should mention that the case $\alpha=1$ is somewhat important even if the attracting measure is degenerate, since is related to the noncommutative weak law of large numbers $[2,6]$.

If the attracted measure has finite variance, we can provide a stronger result.

Proposition 5.7. The only possible non-degenerate limit of the weighted sum

$$
Z_{n}=\frac{X_{1}+\ldots+X_{n}}{B_{n}}-A_{n},
$$

of f.i.d. random variables with non-degenerate common distribution with finite variance, is the semicircle law. In this case

$$
\lim _{n \rightarrow \infty} \frac{\sqrt{n}}{B_{n}}=l<\infty \text {. }
$$

Proof. Easily follows from Maassen Central Limit Theorem [7].

We have then a complete correspondence with the classical probability theory $($ see $[4,5])$. 
It was shown in Theorem 5.5, that the constants $B_{n}$ determining attraction to a stable law of exponent $\alpha$ are necessarily of the form

$$
B_{n}=n^{\frac{1}{\alpha}} h(n)
$$

where $h(n)$ is slowly varying. The most interesting case, for convergence to the semicircle law, is that in which

$$
B_{n}=k n^{\frac{1}{\alpha}}
$$

for $k>0$ constant. On the other hand, any stable law of exponent $\alpha$ belongs to its own domain of attraction, with $B_{n}=k n^{\frac{1}{\alpha}}$. This suggests the following definition.

Definition 5.8. A distribution belongs to the normal domain of attraction of a stable law of exponent $\alpha$ if the normalizing constants are given by

$$
B_{n}=k n^{\frac{1}{\alpha}}
$$

for $k>0$ constant.

In the following we want to apply the results we obtained to the case of f.i.d. random variables with a $\boxplus$-infinitely divisible distribution.

Theorem 5.9. In order that a $⿴$-infinitely divisible law belong to the normal domain of attraction of the semicircle law, it is necessary and sufficient that it have finite variance.

Proof. The sufficiency follows from the Maassen Central Limit Theorem [7]. To prove the necessity, let $\left\{X_{n}\right\}_{n=1}^{\infty}$ be a sequence of f.i.d. random variables in a $W^{*}$-probability space with common distribution $\nu$. By Theorem 4.2 , there exist $a \in \mathbf{R}$ and a positive measure $\sigma$ such that

$$
\phi_{\nu}(z)=a+\int_{-\infty}^{+\infty} \frac{1+t z}{z-t} d \sigma(t) .
$$

Suppose that there exist $k>0$ and a sequence $\left\{A_{n}\right\}_{n=1}^{\infty}$ of real numbers such that $Z_{n}$, given by

$$
Z_{n}=\frac{X_{1}+\ldots+X_{n}}{k \sqrt{n}}-A_{n},
$$

converges to the semicircle law $\gamma_{r}$ for some $r$, but

$$
\int_{-\infty}^{+\infty} t^{2} d \nu(t)=\infty
$$

By Proposition 4.3 we have also

$$
\int_{-\infty}^{+\infty} t^{2} d \sigma(t)=\infty
$$

Then, by Proposition 3.2, there exist $\alpha, \beta>0$ such that

$$
\frac{\sqrt{n}}{k} \phi_{\nu}(k \sqrt{n} z)-A_{n} \underset{n \rightarrow \infty}{\longrightarrow} \phi_{\gamma_{r}}(z)=\frac{r^{2}}{4} \frac{1}{z} \text {, }
$$


uniformly on the compact subsets of the truncated cone $\Gamma_{\alpha, \beta}$. In particular, for every $z=x+i y \in \Gamma_{\alpha, \beta}$,

$$
\Im\left(\frac{\sqrt{n}}{k} \phi_{\nu}(k \sqrt{n} z)\right) \underset{n \rightarrow \infty}{\longrightarrow} \Im \phi_{\gamma_{r}}(z)=\frac{r^{2}}{4} \frac{y}{\left(x^{2}+y^{2}\right)^{\frac{1}{2}}} .
$$

So, if $x=0$, we have that

$$
\Im\left(\frac{\sqrt{n}}{k} \phi_{\nu}(k \sqrt{n} i y)\right) \underset{n \rightarrow \infty}{\longrightarrow} \frac{r^{2}}{4} \frac{1}{y} .
$$

On the other hand,

$$
\begin{aligned}
\lim _{n \rightarrow \infty}\left|\Im\left(\frac{\sqrt{n}}{k} \phi_{\nu}(k \sqrt{n} i y)\right)\right| & =\lim _{n \rightarrow \infty} \frac{n}{k} \int_{-\infty}^{+\infty} \frac{k y\left(1+t^{2}\right)}{t^{2}+n k^{2} y^{2}} d \sigma(t) \\
& =\lim _{n \rightarrow \infty} \int_{-\infty}^{+\infty} \frac{y\left(1+t^{2}\right)}{\frac{t^{2}}{n}+k^{2} y^{2}} d \sigma(t) \\
& =\frac{1}{k^{2} y} \int_{-\infty}^{+\infty}\left(1+t^{2}\right) d \sigma(t)=\infty,
\end{aligned}
$$

by the Monotone Convergence Theorem.

\section{ACKNOWLEDGMENTS}

The author would like to thank Professor Bercovici for encouragement and helpful discussion during the course of the investigation.

\section{REFERENCES}

1. H. Bercovici and D. Voiculescu, Free convolution of measures with unbounded support, Indiana Univ. Math. J. 42 (1993), 733-773.

2. H. Bercovici and V. Pata, The law of large numbers for free identically distributed variables, Preprint, 1995.

3. H. Bercovici and D. Voiculescu, Lévy-Hinčin type theorems for multiplicative and additive free convolution, Pacific J. Math. 153 (1992), 217-248.

4. W. Feller, An introduction to probability theory and its applications, Wiley, New York, 1971.

5. I.A. Ibragimov and Yu.V. Linnik, Independent and stationary sequences of random variables, Wolters-Noordhoff, Groningen, 1971.

6. J.M. Lindsay and V. Pata, Some weak laws of large numbers in non-commutative probability, Preprint, 1994.

7. H. Maassen, Addition of freely independent random variables, J. Funct. Anal. 106 (1992), 409-438.

8. F.J. Murray and J. von Neumann, On rings of operators, Ann. of Math. 37 (1936), 116-229.

9. D. Voiculescu, Addition of certain noncommuting random variables, J. Funct. Anal. 66 (1986), 323-346.

10. D. Voiculescu, Limit laws for random matrices and free products, Invent. Math. 104 (1991), 201-220.

11. D. Voiculescu, K. Dykema, and A. Nica, Free random variables, CRM Monograph Series, vol. 1, Amer. Math. Soc., Providence, RI, 1992.

Department of Mathematics, Indiana University, Bloomington, Indiana 47405

E-mail address: vpata@ucs.indiana.edu 